\title{
Video Article \\ Stereotactically-guided Ablation of the Rat Auditory Cortex, and Localization of the Lesion in the Brain
}

\author{
Verónica Lamas ${ }^{1,2}$, Sheila Estévez ${ }^{1}$, Marianni Pernía ${ }^{1}$, Ignacio Plaza ${ }^{1}$, Miguel A. Merchán ${ }^{1}$ \\ ${ }^{1}$ Institute of Neuroscience of Castilla y León, University of Salamanca \\ ${ }^{2}$ Eaton-Peabody Laboratory, Massachusetts Eye and Ear Infirmary, Department of Otolaryngology, Harvard Medical School
}

Correspondence to: Verónica Lamas at veronica_lamas@meei.harvard.edu

URL: https://www.jove.com/video/56429

DOI: doi: $10.3791 / 56429$

Keywords: Behavior, Issue 128, Auditory cortex, stereotactic location, surgical exposure of the auditory cortex, auditory cortex ablation, localization of cortical lesions, coordinate map

Date Published: 10/11/2017

Citation: Lamas, V., Estévez, S., Pernía, M., Plaza, I., Merchán, M.A. Stereotactically-guided Ablation of the Rat Auditory Cortex, and Localization of the Lesion in the Brain. J. Vis. Exp. (128), e56429, doi:10.3791/56429 (2017).

\section{Abstract}

The rat auditory cortex $(\mathrm{AC})$ is becoming popular among auditory neuroscience investigators who are interested in experience-dependence plasticity, auditory perceptual processes, and cortical control of sound processing in the subcortical auditory nuclei. To address new challenges, a procedure to accurately locate and surgically expose the auditory cortex would expedite this research effort. Stereotactic neurosurgery is routinely used in pre-clinical research in animal models to engraft a needle or electrode at a pre-defined location within the auditory cortex. In the following protocol, we use stereotactic methods in a novel way. We identify four coordinate points over the surface of the temporal bone of the rat to define a window that, once opened, accurately exposes both the primary (A1) and secondary (Dorsal and Ventral) cortices of the AC. Using this method, we then perform a surgical ablation of the AC. After such a manipulation is performed, it is necessary to assess the localization, size, and extension of the lesions made in the cortex. Thus, we also describe a method to easily locate the AC ablation postmortem using a coordinate map constructed by transferring the cytoarchitectural limits of the AC to the surface of the brain. The combination of the stereotactically-guided location and ablation of the AC with the localization of the injured area in a coordinate map postmortem facilitates the validation of information obtained from the animal, and leads to a better analysis and comprehension of the data.

\section{Video Link}

The video component of this article can be found at https://www.jove.com/video/56429/

\section{Introduction}

The rat is one of the most commonly used animal models in auditory neuroscience. The robustness of its behavior makes it able to work for hundreds of trials per day. Its sensitivity and spectral acuity for hearing ${ }^{1,2}$, and the anatomical and functional organization of its central system, comparable to other mammals ${ }^{3}$, make the rat a suitable animal model for analyzing a wide range of research topics in auditory neuroscience. The rat auditory cortex (AC), in particular, has been the subject of several anatomical and physiological studies that have tried to understand its structure, organization, and role in sound processing ${ }^{3}$. Nowadays, the AC has become popular among neuroscientists interested in experiencedependence plasticity, auditory perception, the synaptic basis of receptive field organization, and the cortical control of the sound processing in the subcortical auditory nuclei $i^{4,5,6,7,9}$. To address the challenges that these new approaches pose, procedures that can accurately locate and surgically expose the AC will expedite research efforts. Stereotactic techniques make it easy to localize specific regions within the brain without physiology testing. Although brain size varies slightly between animals, the location of any brain area can be determined using stereotactic coordinates set from landmarks on the skull of the rat brain.

The restricted ablation of the $\mathrm{AC}$ is the surgical removal of the sensory region of the cortex most directly related with hearing. In contrast to other methods used to block the activity of the AC, such as cooling or local lidocaine injections ${ }^{10,11,12}$, the surgical ablation of the AC results in the chronic loss of function. Thus, AC ablations are more suitable for studying the long-term effects of cortical deprivation, as well as the subsequent phenomena of lesion plasticity. The combination of stereotactic methods with surgical ablations of the AC has been used successfully to study the physiological, behavioral, and molecular effects of cortical control deprivation ${ }^{13,14,15,16,17,18,19}$. For example, a rat model with bilateral AC ablations has been used to study the effects of cortical ablation in the auditory startle reflex and auditory brainstem responses (ABR) ${ }^{16}$. Recently, we have compared the effects that unilateral versus bilateral ablations of the rat AC produce in ABR thresholds, amplitudes, and latencies at different time points after the injury ${ }^{18}$. In addition, the rat model of restrictive AC ablation has also been used to study the effect of corticofugal pathway degeneration in the inferior collicus ${ }^{13,14,15}$ and the inner ear ${ }^{17,19}$. After such a manipulation is performed in the brain, it is necessary to assess the localization, size, and extension of the lesions made in the cortex. Although very useful, the main limitation of tonotopic maps based on neuronal response ${ }^{20,21}$ are the electrophysiological techniques required to locate the auditory fields in the rat brain. Since not all laboratories have the necessary equipment and/or expertise to make such recordings, we constructed a coordinate map based on the transfer of the cytoarchitectural limits of the AC to an image of the brain's surface ${ }^{18}$. This map can be very useful to locate the AC without physiology testing. 
The present protocol describes a method for the stereotactically guided location, surgical exposure, and ablation of the AC in rats. It also describes how to use our coordinate map ${ }^{18}$ to easily localize the extension of the lesion over a picture of the surface of the ablated brains.

\section{Protocol}

This study was carried out in strict accordance with both Spanish regulations (Royal Decree 53/2013 - Law 32/2007) and European Union guidelines (Directive 2010/63/EU) on the care and use of animals in biomedical research.

\section{Preparation of the Rat}

NOTE: We performed the experiments in male rats to avoid any hormonal changes.

1. Anesthetize the animal using a mix of ketamine hydrochloride $(30 \mathrm{mg} / \mathrm{kg})$ and xylazine hydrochloride $(5 \mathrm{mg} / \mathrm{kg})$ injected intramuscularly; with this dose, the rat should be deeply anesthetized for around $1 \mathrm{~h}$.

2. Pinch the rat's toe; absence of a withdrawal reflex indicates that the animal is fully unconscious. If the rat responds to the pinch, give supplementary anesthesia at one third of the initial dose.

3. Shave the scalp and disinfect the surgical area with povidone-iodine.

4. Place the animal on a heating pad to maintain a temperature of $38{ }^{\circ} \mathrm{C}$ and stabilize the animal's head in a stereotaxic frame using two ear bars and a bite bar. Use caution to avoid piercing the tympanic membrane with the ear bars.

5. Protect the eyes by applying a drop of ophthalmic gel or serum saline to each eye.

\section{Location of the $A C$ in the Temporal Bone of the Rat}

1. Using a scalpel, make an incision along the midline to expose the skull and retract the periosteum covering the surface of the cranium.

2. Use a sterile cotton tip to gently remove any blood covering the surface of the skull to visualize bregma, lambda, and interaural 0 according to the Paxinos and Watson atlas of the rat brain ${ }^{22}$.

3. Make an incision in the temporal muscle near its dorsal insertion on the skull with a scalpel. Pull the muscle out using a needle and suture material, and fix the suture material to the stereotactic frame; this will expose the temporal bone. If bleeding occurs, rinse with cold sterile saline.

4. Place a sterile straight needle in the stereotactic micromanipulator, making sure that it is fully secured.

5. Slowly lower the needle until it is right above the surface of the skull, so that the tip of the needle is set to interaural 0 . Set this point as zero, and determine coordinates from this point.

6. Depending on the brain area of interest, vary the stereotactic coordinates. Determine these coordinates by utilizing the Paxinos and Watson atlas of the rat brain ${ }^{22}$. Once the coordinates are determined, move the needle to match those coordinates.

7. Target the $A C$ using the coordinates of the following four points: $A: A / P=-5.8 \mathrm{~mm}, M / L=+/-6.4 \mathrm{~mm} ; B: A / P=-2.7 \mathrm{~mm}, \mathrm{M} / \mathrm{L}=+/-6.4 \mathrm{~mm} ; \mathrm{C}$ : $\mathrm{A} / \mathrm{P}=-2.7 \mathrm{~mm}, \mathrm{M} / \mathrm{L}=+/-8.67 \mathrm{~mm} ; \mathrm{D}: \mathrm{A} / \mathrm{P}=-5.8 \mathrm{~mm}, \mathrm{M} / \mathrm{L}=+/-8.67 \mathrm{~mm}$. Lower the needle to right above the temporal bone to visualize each of these four points. Using a marker pen, mark the points on the temporal bone and connect them in order to draw a rectangle; the rectangle will serve as a guide to open of a window in the bone (Figure 1).

\section{Surgical Exposure of the AC}

1. Open the window using an electric drill and a small drill bit $(0.6 \mathrm{~mm} \varnothing)$. Drill the perimeter of the rectangle at $8,000 \mathrm{rpm}$ until the bone gives away. Cool the drilling surface by rinsing with cold sterile saline to prevent damage to subcortical structures. When the bone gives way, a drop in resistance can be detected. Be careful to not drill the brain.

2. When the borders are loose, pull up the covering bone with fine forceps and store it in cold sterile saline.

\section{Ablation of the AC}

1. Using a surgical microscope (10X), gently cut the meninges with a microsurgical knife and remove them using two fine-pointed forceps. If bleeding occurs, rinse with cold sterile saline.

2. Gently aspirate the $A C$ using a surgical suction device (pressure -0.24 bar) coupled to a sterile $20 \mathrm{G}$ blunt tip needle. This point is critical and needs to be performed very carefully: aspirate only the six cortical layers and not the underlying white matter.

3. Aspirate until the perforating arteries stop bleeding.

4. When the aspiration is finished, cover the injured area with the extracted bone and apply an absorbable hemostatic gauze.

5. Let the temporal muscle recover its original position, and then suture the skin using wound clips (9 mm). Apply antibiotic ointment (see the Table of Materials). Continue to apply ointment to the wound twice daily for three days.

NOTE: Each application consists of a thin layer applied on the wound.

6. Inject buprenorphine subcutaneously in the back of the rat $(0.05 \mathrm{mg} / \mathrm{kg})$ as an analgesic $1 \mathrm{~h}$ after the surgery, and then every $8 \mathrm{~h}$ during $72 \mathrm{~h}$.

7. Keep the animal on the heating pad until it wakes up, and return it to its housing cage to recover.

8. House animals individually to prevent cage mates from touching the sutured area, and provide some enrichment items. Change the sawdust daily to prevent infection, and carefully check that the animal recovers properly and does not show any signs of discomfort.

\section{Tissue Collection}

Caution: When handling paraformaldehyde (PFA), both solid and aqueous, wear personal protective equipment (PPE) and use a safety cabinet. 
NOTE: Prepare $750 \mathrm{~mL}$ formaldehyde solution by dissolving $4 \%(\mathrm{w} / \mathrm{v})$ PFA into $1 x$ phosphate buffered solution (PBS) using heat $\left(55{ }^{\circ} \mathrm{C}\right)$. Filter the formaldehyde solution with filter paper. Prepare Ringer's Solution by dissolving $8.5 \mathrm{~g}$ of NaCl, $0.25 \mathrm{~g}$ of $\mathrm{KCl}$, and $0.2 \mathrm{~g} \mathrm{NaHCO}$ in 1,000 mL of water (solution $\mathrm{pH}=6.9$ )

1. Let the animal survive for as long as it is needed for the study. When the research performed with the AC ablated rat is completed, terminally anaesthetize it by intra-peritoneal injection of $0.1 \mathrm{~mL}$ of sodium pentobarbital $(60 \mathrm{mg} / \mathrm{kg})$. Assess the depth of anesthesia by toe pinch and the absence of withdrawal reflex.

2. When the animal is deeply anesthetized, perform an intracardiac perfusion ${ }^{23}$ of $125 \mathrm{~mL}$ of Ringer's Solution followed by $750 \mathrm{~mL}$ of formaldehyde solution by using a needle gauge of $1.8 \mathrm{~mm}$ of inside diameter.

3. When the perfusion is finished, decapitate the rat at the first cervical vertebra.

4. Use scissors to remove the skin and muscle from the head and expose the skull. Use scissors to cut and open the foramen magnum and remove the back of the skull.

5. Make a transversal cut into the orbital bone using Spencer scissors, and use rongeurs to cut along the top edges of the skull to expose the brain. Be careful not to damage the brain.

6. Once the brain is exposed, carefully remove the dura mater using fine-pointed forceps. Use a finger to gently scoop under and elevate the brain. Raise the brain and cut the nerves until it is free. Immerse the brain in formaldehyde solution and store it at $4{ }^{\circ} \mathrm{C}$ for $24 \mathrm{~h}$.

\section{Localization of the AC Lesions}

1. After post-fixation, carefully place the brain into a sagittal rat brain matrix exposing the lateral surface of the brain.

2. Place a camera $21 \mathrm{~cm}$ above the cortex surface using a camera holder, select the "super macro shooting" mode, and take a picture of the brain surface.

3. Place the brain into a coronal rat brain matrix exposing the dorsal surface of the brain, and take another picture.

4. Using an image editor program, open the images and scale them down $50 \%$ to make it easier to work with them. Identify the bregma, lambda, and interaural 0 references on the picture according to Paxinos and Watson coordinates ${ }^{19}$, and mark their position in the pictures (Figure 2). Draw the contour of the ablation over the lateral picture of the brain. Calculate the perimeter.

5. Import the coordinate map where the primary (A1) and secondary regions (Dorsal and Ventral Cortices) of AC are located ${ }^{18}$ to the file of the editor program where you are working with the pictures. Click on the map and drag it to superimpose it to the lateral photograph of the ablated brain.

1. Make the bregma and lambda references of the coordinate map coincide with the bregma and lambda references identified in the picture of the lateral brain.

2. Use the rhinal fissure as a reference to adjust the picture of the brain to the map, and make them coincide (Figure 2B).

6. Calculate the percentage of the lesion relative to the area occupied by the AC.

\section{Representative Results}

We performed a stereotactically guided location, surgical exposure, and unilateral ablation of the AC in three Wistar rats. The localization of the lesion confirmed that ablations performed in the three rats encroached the major subdivisions of the AC (primary, dorsal, and ventral cortices), and comprised a range of 80 to $100 \%$ of the total AC area (Figure 2B).

The protocol described here to perform restrictive $\mathrm{AC}$ ablations has previously been used in our laboratory to study the long-term effects of cortical control deprivation in the subcortical auditory nuclei, as well as the subsequent phenomena of plasticity. In these studies, the protocol of $\mathrm{AC}$ ablations was validated by applying physiological (ABR), behavioral (startle responses, prepulse inhibition; PPI), and molecular (DNA microarrays, qPCR, and Western Blot) methods ${ }^{13,14,15,16,17,18,19}$. Here, to demonstrate the efficacy of our protocol, we let the three AC ablated rats survive for one week, and collected the cochleae during the tissue collection step to study the changes in the expression of the most relevant AMPA subunits present in the adult cochlea, GluA2 and GluA3, by qPCR. The comparison between the transcripts from AC ablated rats and sham control animals where all the surgery process but not the cortical ablation was performed showed a down-regulation for GluA2, and an up-regulation for GluA3 in both cochleae (Figure 3), which is in agreement with our previous study ${ }^{19}$. 

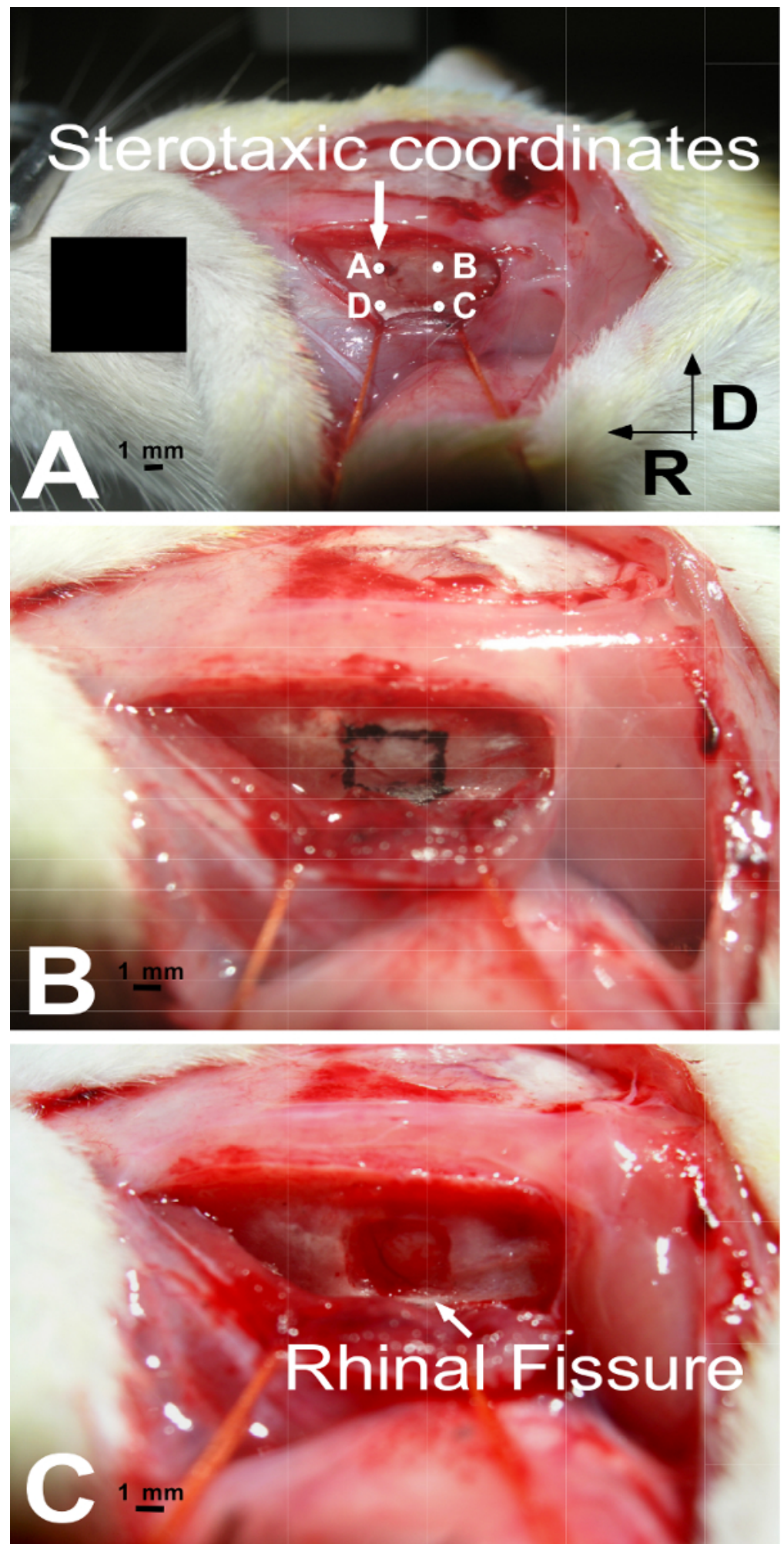

Figure 1: Images of the rat temporal bone at three different surgical steps. (A) Transfer of the stereotaxic coordinates of the AC to the temporal bone. The coordinates of the four points are: $A: A / P=-5.8 \mathrm{~mm}, M / L=+/-6.4 \mathrm{~mm} ; \mathrm{B}: \mathrm{A} / \mathrm{P}=-2.7 \mathrm{~mm}, \mathrm{M} / \mathrm{L}=+/-6.4 \mathrm{~mm} ; \mathrm{C}: \mathrm{A} / \mathrm{P}=-2.7 \mathrm{~mm}$, $M / L=+/-8.67 \mathrm{~mm} ; D: A / P=-5.8 \mathrm{~mm}, M / L=+/-8.67 \mathrm{~mm}$. (B) The coordinates are used as a reference to draw a rectangle on the surface of the temporal bone that will guide the opening of a window. (C) Shows the window opened in the bone after drilling. The meninges with blood vessels can be observed on the surface of the brain. R: rostral, D: dorsal. Please click here to view a larger version of this figure. 


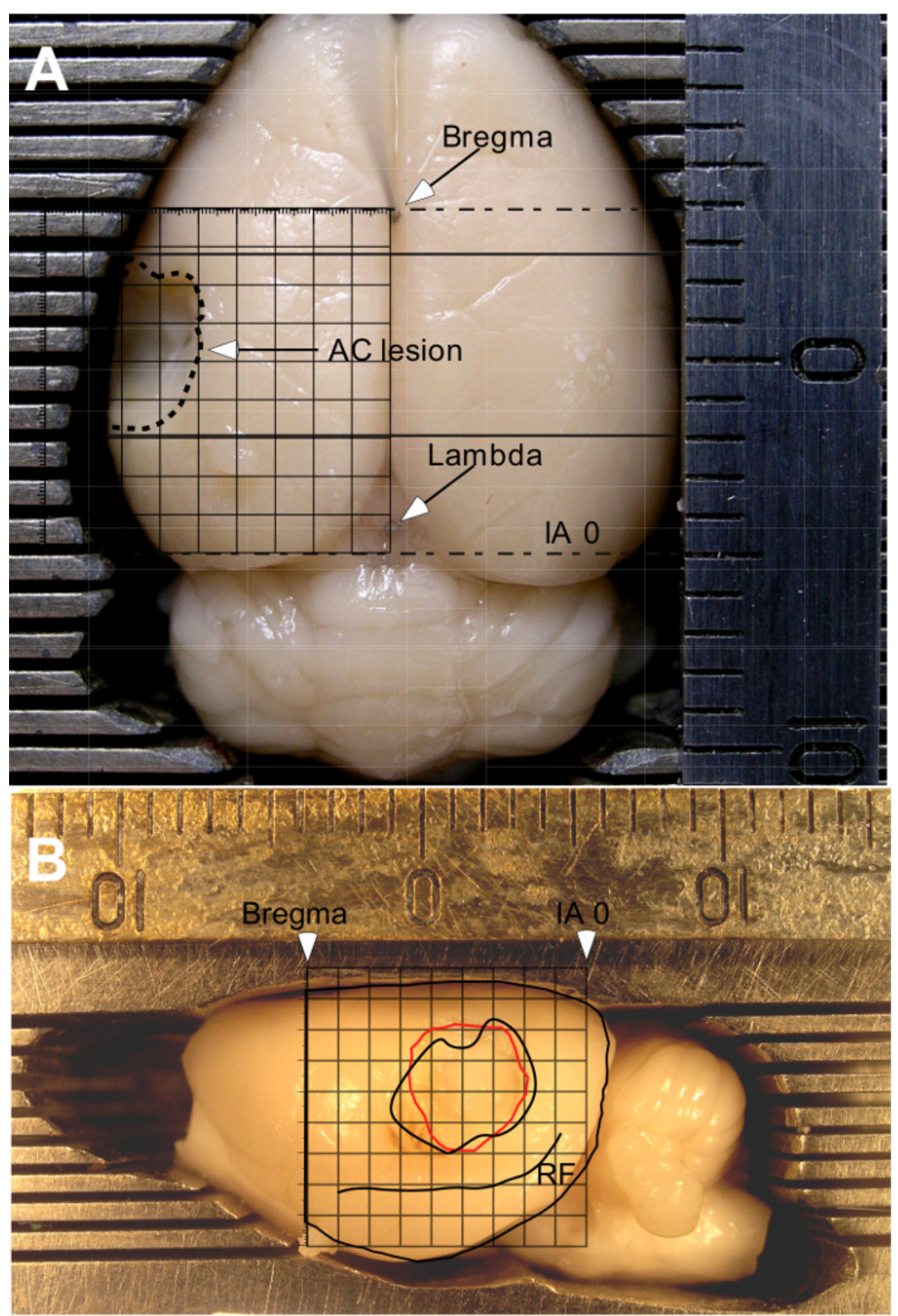

Figure 2: Procedure for locating lesions in the rat brain. (A) Photograph of the dorsal surface of an AC ablated brain with stereotactically implanted needles in Lambda and Bregma (according to Paxinos and Watson coordinates ${ }^{19}$ ). Dotted lines mark the position of Bregma and Interaural 0 in the $9 \mathrm{~cm} \times 9 \mathrm{~cm}$ grid, as well as in the dorsal surface of the brain. (B) Photograph of the lateral surface of the ablated brain superimposed to the coordinate map of the AC. The perimeter of the lesion is labeled in red in the picture. The perimeter of the AC area is labeled in black in the map. In this example, the percentage of AC ablation with respect to the total area occupied by the AC is $84.79 \%$. AC: auditory cortex, IA: inter aural, FR: Rhinal Fissure. Please click here to view a larger version of this figure. 


\section{mRNA levels of AMPA receptor subunits 7 days after AC ablation}

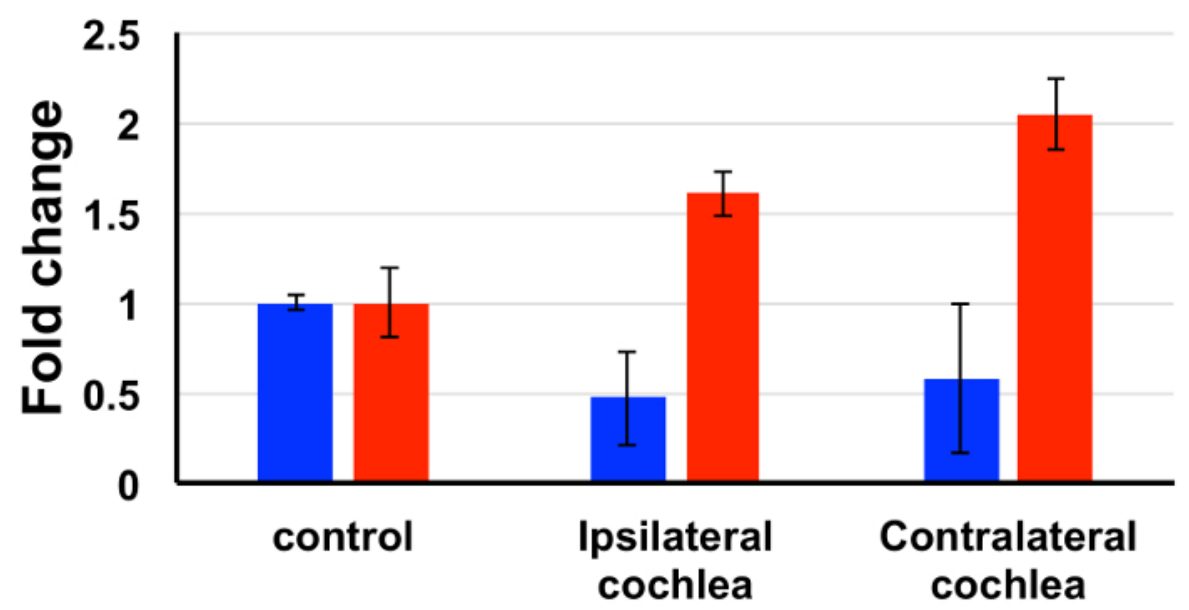

- GIuA2 GIuA3

Figure 3: Changes in mRNA levels of AMPA receptor subunits GluA2 and GluA3 after unilateral AC ablations 7 days post-lesion. Results are presented as the mean \pm standard deviation of the fold change. Changes for GluA2 transcripts are represented in blue. Changes for GluA3 transcripts are presented in red. A significant decrease in GluA2 and an increase in GluA3 is observed in both cochleae (ipsi- and contralateral to the ablation) relative to sham controls with no cortical ablations at 7 days after the surgery; this is in agreement with our previous results ${ }^{19}$. Please click here to view a larger version of this figure.

\section{Discussion}

A successful brain surgery hinges upon two factors: keeping the animal alive during and after the procedure, and accurately locating the area of interest. Ensuring that the rat is deeply anesthetized during the surgery (testing the withdrawal reflex), and receives adequate analgesics and non-ototoxic antibiotics should help survival. Additionally, the rat should be kept on a heating pad until it wakes from anesthesia to avoid hypothermia. Suturing will decrease the susceptibility to infection, and proper technique is vital: Animals will pick at their wound clips, so they should be implanted tight enough to prevent removal without placing too much tension on the wound.

To accurately locate the AC (or any other cortical area), it is important to determine the position of bregma, lambda, and interaural 0 to use them as references to calculate the limits of the targeted region. Any mistake in calculating the coordinates will result in the partial ablation of AC or the undesired aspiration of other surrounding areas. Thus, the needle tip should only touch the bone at interaural 0 , and then translate the anteroposterior and medio-lateral coordinates according to what is described in this protocol.

In this manuscript, we have also described how to surgically expose and ablate the AC. There are three critical steps: the drilling process, the opening and removal of the meninges, and the ablation by aspiration. Drilling should be performed at a low speed with minimum pressure, as a high drilling speed generates heat that can affect nearby subcortical structures. However, maintaining a low speed and cooling the drilling area with cold sterile saline should prevent any damage. In addition, minimum pressure is essential to avoid a sudden break of the skull and subsequent injury to the underlying cortex. The opening and removal of the meninges that cover the AC should be performed carefully to avoid breaking the blood vessels. If bleeding occurs, the early and late prognosis is generally unfavorable and it is questionable whether such an animal meets the inclusion criteria for a reliable study. We recommend euthanasia in this case. Finally, the aspiration (probably the most difficult aspect in performing an effective lesion), must be restricted to the gray matter. There are two indicators that can help detect the presence of the white matter: (1) a change in the color contrast, as the white matter is brighter than the grey matter; and (2) the cessation of bleeding from the perforating arteries.

After any manipulation performed in the brain, it is necessary to assess the localization, size, and extension of the procedure made in the cortex for the subsequent analysis and validation of data obtained from the animal. In this manuscript, we detail how to localize the ablation performed in the cortex using a coordinate map previously described by our group ${ }^{18}$. This map was constructed using anatomical references obtained from serial section reconstructions of histological sections, correlated with the Paxinos and Watson atlas of the rat brain ${ }^{22}$. Accordingly, the map differentiates between the primary (A1) and secondary cortices (Dorsal and Ventral) of the AC. The main advantage of this coordinate map is that it allows the rapid localization of the lesion by superimposing a picture taken from the lateral surface of the brain placed in a sagittal brain matrix. Another advantage is that laboratories with less experience in anatomy can use the map by adapting it to their animal models. It is only necessary to set the distances between bregma, lambda, and interaural 0 references in a control perfused brain, and scale the map up or down accordingly. Use the Rhinal Fissure as reference to adjust the picture of the brain to the map. The depth of the ablation cannot be determined in this coordinate map, so it should be determined in brain histological sections.

The combination of stereotactic methods with the surgical exposure of the AC are basic methods that could be easily adapted by any investigator who wishes to target the AC in the rat. This might be for an acute experiment or one that requires implantation of permanent devices. Moreover, the surgical ablation of the $A C$ has been previously used as a model to study the effects of chronic cortical deprivation in hearing. AC ablations 
could also be used to study the effects that unilateral AC ablations exert in other cortical areas, or serve as a model of stroke. Thus, the experimental designs described here are useful methods that can be applied individually or in combination to a wide range of experimental designs.

\section{Disclosures}

The authors declare that the research was conducted in the absence of any commercial or financial relationships that could be construed as a potential conflict of interest.

\section{Acknowledgements}

This research was supported by a grant from the Ministry of Economy and Competitiveness (MINECO) of the Government of Spain, SAF2016-78898-C2-2-R.

\section{References}

1. Talwar, S. K., \& Gerstein, G. L. Auditory frequency discrimination in the white rat. Hear Res. 126 (1-2), 135-150 (1999).

2. Heffner, H. E., Heffner, R. S., Contos, C., \& Ott, T. Audiogram of the hooded Norway rat. Hear Res. 73(2), 244-247 (1994).

3. Malmierca, M. S., \& Merchán, M. A. Auditory System. The Rat Nervous System. 995-1080. Academic Press. San Diego. (2004).

4. Delano, P. H., \& Elgoyhen, A. B. Editorial: Auditory Efferent System: New Insights from Cortex to Cochlea. Front Syst Neurosci. 10 (June), 1-2 (2016).

5. Dinse, H. R. Sound Case for Enrichment. Focus on "Environmental Enrichment Improves Response Strength, Threshold, Selectivity, and Latency of auditory cortex Neurons." J Neurophysiol. 92(1), 36-37 (2004).

6. Polley, D. B., Heiser, M. A., Blake, D. T., Schreiner, C. E., \& Merzenich, M. M. Associative learning shapes the neural code for stimulus magnitude in primary auditory cortex. Proc Natl Acad Sci U S A. 101(46), 16351-16356 (2004).

7. Kaur, S. Intracortical Pathways Determine Breadth of Subthreshold Frequency Receptive Fields in Primary auditory cortex. $J$ Neurophysiol. 91(6), 2551-2567 (2004).

8. Talwar, S. K., Musial, P. G., \& Gerstein, G. L. Role of mammalian auditory cortex in the perception of elementary sound properties. $J$ Neurophysiol. 85(6), 2350-2358 (2001).

9. Tan, A. Y. Y., Atencio, C. A., Polley, D. B., Merzenich, M. M., \& Schreiner, C. E. Unbalanced synaptic inhibition can create intensity-tuned auditory cortex neurons. Neuroscience. 146(1), 449-462 (2007).

10. León, A., Elgueda, D., Silva, M. A., Hamamé, C. M., \& Delano, P. H. Auditory cortex basal activity modulates cochlear responses in chinchillas. PLOS ONE. 7(4), e36203 (2012).

11. Jager, K., \& Kossl, M. Corticofugal Modulation of DPOAEs in Gerbils. Hear Res. 332, 61-72 (2016).

12. Dragicevic, C. D. et al. The Olivocochlear Reflex Strength and Cochlear Sensitivity are Independently Modulated by auditory cortex Microstimulation. J Assoc Res Otolaryngol. 16(2), 223-240 (2015).

13. Clarkson, C., Herrero-Turrión, M. J., \& Merchán, M. A. Cortical Auditory Deafferentation Induces Long-Term Plasticity in the Inferior Colliculus of Adult Rats: Microarray and qPCR Analysis. Front Neural Circuits. 6, 86 (2012).

14. Clarkson, C., Juíz, J. M., \& Merch́an, M. A. Long-term regulation in calretinin staining in the rat inferior colliculus after unilateral auditory cortical ablation. J Comp Neurol. 518, 4261-4276 (2010).

15. Clarkson, C., Juíz, J. M., \& Merchán, M. A. Transient down-regulation of sound-induced c-Fos protein expression in the inferior colliculus after ablation of the auditory cortex. Front Neuroanat. 4, 141, (2010).

16. Hunter, K. P., \& Willott, J. F. Effects of bilateral lesions of auditory cortex in mice on the acoustic startle response. Physiol Behav. 54(6), 1133-9, (1993).

17. Lamas, V., Arevalo, J. C., Juiz, J. M., \& Merchán, M. A. Acoustic input and efferent activity regulate the expression of molecules involved in cochlear micromechanics. Front Syst Neurosci. 8, 253, (2014).

18. Lamas, V., Alvarado, J. C., Carro, J., \& Merchán, M. A. Long-term evolution of brainstem electrical evoked responses to sound after restricted ablation of the auditory cortex. PLOS ONE. 8(9), e73585 (2013).

19. Lamas, V., Juiz, J. M., \& Merchán, M. A. Ablation of the auditory cortex results in changes in the expression of neurotransmission-related mRNAs in the cochlea. Hear Res. 346, 71-80 (2017).

20. Doron, N. N., Ledoux, J. E., \& Semple, M. N. Redefining the tonotopic core of rat auditory cortex: physiological evidence for a posterior field. J Comp Neurol. 453(4), 345-60 (2002).

21. Polley, D. B., Read, H. L., Storace, D. a \& Merzenich, M. M. Multiparametric auditory receptive field organization across five cortical fields in the albino rat. $J$ Neurophysiol. 97(5), 3621-3638 (2007).

22. Paxinos, G., \& Watson, C. The Rat Brain in Stereotaxic Coordinates. Academic Press. Sydney. (2005).

23. Gage, G. J., \& Kipke, D. R. Whole Animal Perfusion Fixation for Rodents. J Vis Exp. (65), e3564 (2012). 